\title{
MicroRNA-99a induces G1-phase cell cycle arrest and suppresses tumorigenicity in renal cell carcinoma
}

$\mathrm{Li} \mathrm{Cui}^{1 \dagger}$, Hua Zhou ${ }^{2+}$, Hu Zhao ${ }^{3 \dagger}$, Yaojun Zhou ${ }^{1}$, Renfang Xu', Xianlin Xu' ${ }^{1}$, Lu Zheng ${ }^{4}$, Zhong Xue ${ }^{1}$, Wei Xia ${ }^{1}$, Bo Zhang ${ }^{1}$, Tao Ding ${ }^{1}$, Yunjie Cao', Zinong Tian' , Qianqian Shi ${ }^{1}$ and Xiaozhou He ${ }^{1 *}$

\begin{abstract}
Background: A growing body of evidence suggests that microRNAs (miRNAs) play an important role in cancer diagnosis and therapy. MicroRNA-99a (miR-99a), a potential tumor suppressor, is downregulated in several human malignancies. The expression and function of miR-99a, however, have not been investigated in human renal cell carcinoma (RCC) so far. We therefore examined the expression of miR-99a in RCC cell lines and tissues, and assessed the impact of miR-99a on the tumorigenesis of RCC.
\end{abstract}

Methods: MiR-99a levels in 40 pairs of RCC and matched adjacent non-tumor tissues were assessed by real-time quantitative Reverse Transcription PCR (qRT-PCR). The RCC cell lines 786-O and OS-RC-2 were transfected with miR-99a mimics to restore the expression of miR-99a. The effects of miR-99a were then assessed by cell proliferation, cell cycle, transwell, and colony formation assay. A murine xenograft model of RCC was used to confirm the effect of miR-99a on tumorigenicity in vivo. Potential target genes were identified by western blotting and luciferase reporter assay.

Results: We found that miR-99a was remarkably downregulated in RCC and low expression level of miR-99a was correlated with poor survival of RCC patients. Restoration of miR-99a dramatically suppressed RCC cells growth, clonability, migration and invasion as well as induced G1-phase cell cycle arrest in vitro. Moreover, intratumoral delivery of miR-99a could inhibit tumor growth in murine xenograft models of human RCC. In addition, we also fond that mammalian target of rapamycin (mTOR) was a direct target of miR-99a in RCC cells. Furthermore, siRNA-mediated knockdown of mTOR partially phenocopied the effect of miR-99a overexpression, suggesting that the tumor suppressive role of miR-99a may be mediated primarily through mTOR regulation.

Conclusions: Collectively, these results demonstrate for the first time, to our knowledge, that deregulation of miR-99a is involved in the etiology of RCC partially via direct targeting mTOR pathway, which suggests that miR-99a may offer an attractive new target for diagnostic and therapeutic intervention in RCC.

Keywords: MicroRNA-99a, mTOR, Renal cell carcinoma

\section{Background}

Renal cell carcinoma (RCC) is the most common neoplasma of the kidney in adults accounting for about 3\% of adult malignancies [1], with having the highest mortality rate at over $40 \%$ [2]. The 5-year survival of RCC is estimated to be approximately 55\% [3], and that of

\footnotetext{
* Correspondence: clturtle@126.com

${ }^{\dagger}$ Equal contributors

'Department of Urology, The Third Affiliated Hospital of Soochow University, 185 Juqian Street, Changzhou 213003, China

Full list of author information is available at the end of the article
}

metastatic RCC is approximately 10\% [4]. Surgical resection is still the only definitive treatment for RCC, but after the curative nephrectomy, $20-40 \%$ patients will develop recurrence [5]. This is mainly a consequence of the fact that RCC is resistant to both chemotherapy and radiotherapy [6]. So no adjuvant therapy is available in clinical routine. Moreover, the absence of biomarkers for early detection and follow-up of the disease complicate the on-time diagnosis. Therefore, novel tumor markers that have higher sensitivity and reliability and effective therapeutic methods are urgently needed for RCC.

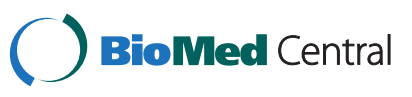


MicroRNAs (miRNAs) are a class of naturally occurring, non-coding, short single stranded RNAs, in the size range 19-25 nucleotides, that regulate gene expression at the post-transcriptional level, by binding through partial sequence homology, to the $3^{\prime}$ untranslated region (3/UTR) of mammalian target mRNAs and causing translational inhibition and/or mRNA degradation [7]. It has been firmly established that miRNAs control various key cellular processes, such as proliferation, cell cycle, differentiation, and tumorigenesis [8]. In recent years, numerous studies have shown aberrant expression of miRNAs in human cancers [9], including RCC [10], some of which function as tumor suppressor genes or oncogenes [11]. Due to their tissue- and disease-specific expression patterns and tremendous regulatory potential, miRNAs are being identified as diagnostic and prognostic cancer biomarkers, as well as additional therapeutic tools [12].

It has been reported that miR-99a is transcribed from the commonly deleted region at $21 \mathrm{q} 21$ in human lung cancers [13], and that miR-99a is downregulated in ovarian carcinoma [14], squamous cell carcinoma of the tongue [15], squamous cell lung carcinoma [16], hepatocellular carcinoma [17], bladder cancer [18], prostate cancer [19] and childhood adrenocortical tumors [20]. These findings indicate that miR-99a is widely downregulated in human cancers, suggesting a potential role of miR-99a as a tumor suppressor. However, up to date, there are no studies of miR-99a in RCC. Thus, we concentrated on miR-99a in RCC.

The present study was undertaken to examine the expression of miR-99a in RCC cell lines and tissues, assess the impact of miR-99a on RCC cells and RCC xenograft modle, and identify target genes for miR-99a that might mediate their biological effects. In this study, we observed that miR-99a was remarkably downregulated in RCC cell lines and tissues and correlated with overall survival of RCC patients. Restoration of miR-99a induced G1-phase cell cycle arrest in vitro and dramatically suppressed tumorigenicity of RCC in vitro and in vivo. In addition, with the help of a bioinformatic analysis, we found that the mammalian target of rapamicin (mTOR), a key promoter of cell growth, was a direct target of miR-99a in RCC cells. Furthermore, siRNA-mediated knockdown of mTOR partially phenocopied miR-99a restoration suggesting that the tumor suppressive role of miR-99a may be mediated primarily through mTOR regulation. Our study suggests that miR-99a may offer an attractive new target for diagnostic and therapeutic intervention in RCC.

\section{Methods}

\section{Tissue samples}

The study was approved by the ethics committee of the Third Affiliated Hospital of Soochow University. Written informed consent was obtained from each patient for the use of material to research purposes. All tissue samples (40 pairs) contained more than $80 \%$ tumor cells were obtained from the Department of Urology, the Third Affiliated Hospital of Soochow University, China. Tumor tissues were harvested during partial or radical nephrectomy and confirmed renal cell carcinoma by pathological study post operatively. Adjacent non-tumor tissues were also resected simultaneously, and half of them were sent for pathological inspection to rule out contamination of tumor. Tissue samples were immediately frozen in liquid nitrogen until analysis.

\section{Cell lines and cell culture}

The nonmalignant SV-40 immortalized renal cell line HK-2 was obtained from KeyGen Biotech (Nanjing, China), which was maintained in DMEM with $10 \%$ FBS. The human renal cancer cell lines 786-0 and OS-RC-2 were obtained from the Chinese Academy of Sciences Cell Bank, which were maintained in RPMI 1640 with $10 \%$ FBS. All cell lines were cultured at $37^{\circ} \mathrm{C}$ in a humidified incubator $\left(5 \% \mathrm{CO}_{2}\right)$.

\section{miRNA/siRNA transfections}

2'-O-methyl (2'-O-Me) oligonucleotides were chemically synthesized by GenePharma Biotechnology (Shanghai, China). The sequences were as follows: miR-99amimics: (forward) 5'-AACCCGUAGAUCCGAUCUUGUG-3',(reverse) 5'-CA AGAUCGGAUCUACGGGUUUU-3'; mTOR-siRNA: (forward) 5'-ACCAUGAACCAUGUCCUAAGCUGTG-3', (reverse) 5'-CACAGCUUAGGACAUGGUUCAUGGUAU-3'; negative control (NC) : (forward) 5'-UUCUCCGAACGUGUC ACGUTT-3', (reverse) 5'-ACGUGACACGUUCGGAGAA TT-3'. Cells at 70\%-80\% confluence were transfected with miR-99a mimics, mTOR-siRNA or negative control (NC) using Lipofectamine 2000 (Invitrogen) according to the manufacturer's protocol.

\section{RNA isolation and real-time qRT- PCR}

Total RNAs were isolated from RCC tissues and cell lines using TRIzol reagent (Invitrogen, USA) for miRNA analyses. MiR-99a real-time qRT-PCR was performed by the TaqMan miRNA assays (Applied Biosystems, USA) and U6 was used as an internal control. PCR cycles were as follows: initial denaturation at $95^{\circ} \mathrm{C}$ for 10 minutes, followed by 40 cycles at $95^{\circ} \mathrm{C}$ for 15 seconds and $60^{\circ} \mathrm{C}$ for 1 minute. The relative miRNA expression was calculated using the $2^{-\Delta \Delta \mathrm{Ct}}$ method.

\section{Cell proliferation assay}

786-0 and OS-RC-2 cells were transfected with the miR-99a mimics, mTOR-siRNA or negative control (NC) for 48 hours and then seeded at 2000 cells per well in 96-well plates. $10 \mu \mathrm{l}$ CCK-8 solution was added to 
$100 \mu \mathrm{l}$ culture media per well above and then the plate was incubated for at $37^{\circ} \mathrm{C} 1.5$ hours. The absorbance was measured at $450 \mathrm{~nm}$ using a Vmax microplate spectrophotometer (Molecular Devices, Sunnyvale, CA). Each sample was assayed in triplicate. This procedure was repeated at 24, 48, 72 and 96 hours after transfection.

\section{Colony formation assay}

786-O and OS-RC-2 cells were transfected with the miR-99a mimics, mTOR-siRNA or negative control (NC) for 24 hours and then seeded for colony formation in 6-well plates at 200 cells per well. After 15 days, cells were stained with Giemsa, and then colonies were counted only if a single clone contained more than 100 cells. Each assay was performed in triplicate.

\section{Cell cycle assay}

Transfected RCC cells in the log phase of growth were collected and fixed in $75 \%$ ethanol at $-20^{\circ} \mathrm{C}$ for 16 hours. For cell cycle analysis, transfected cells were stained with propidium iodide and examined with a fluorescenceactivated cell sorting (FACS) flow cytometer (BD Biosciences, San Jose,CA), and DNA histograms were analyzed with modified software. Each test was repeated in triplicate.

\section{Cell migration and invasion}

786-O and OS-RC-2 cells were transfected with the miR-99a mimics, mTOR-siRNA or negative control (NC), cultivated for 48 hours, and transferred on the top of Non-matrigel-coated/ Matrigel-coated chambers (24well insert, $8-\mu \mathrm{m}$ pore size, BD Biosciences, San Jose, USA) in a serum-free RPMI 1640 and the medium containing 30\% fetal calf serum was added to the lower chamber as a chemoattractant. After incubation for 48 hours, non-migrated/non-invaded cells were removed from the upper well with cotton swabs while the migrated/invaded cells were then fixed with $4 \%$ paraformaldehyde, stained with $0.1 \%$ crystal violet, and photographed $(\times 200)$ in five independent fields for each well. Each test was repeated in triplicate.

\section{Nude mouse tumor xenograft model}

All experimental procedures involving the use of animals were in accordance with the Guide for the Care and Use of Laboratory Animals and were approved by the ethics committee of the Third Affiliated Hospital of Soochow University. Nude mice (5- to 6-week old; SLAC ANIMAL, China; $\mathrm{n}=12$ ) received subcutaneous injections of $3 \times 10^{6} 786-0$ cells in the right flank area in a volume of $200 \mu \mathrm{l}$. Once palpable tumors developed, the volume of tumor was measured with a caliper every 4 days, using the formula: volume $=\left(\right.$ length $\times$ width $\left.^{2}\right) / 2$. When the tumor volume reached an average volume of 75 to $100 \mathrm{~mm}^{3}$, the mice were randomly divided into two groups (six mice per group). These mice were then treated with 200 pmol miR-99a or NC mimics in $10 \mu \mathrm{l} \mathrm{Lipo-}$ fectamine 2000 through a local injection of the xenograft tumor at multiple sites.

\section{Luciferase activity assay}

The portion of the $3^{\prime} \mathrm{UTR}$ region (908 bp) of human mTOR gene containing the miR-99a binding site was amplified by PCR using the following primers: mTOR3'UTR-F: 5'-CTTTCAGAAACTGGAGGCCCAG-3' and mTOR-3'UTR-R: 5'-TGGTGTCTAGACATGGCTACAC TTTATAC-3'. This portion was amplified and cloned into the $\mathrm{XbaI}$ site of the pGL3-control vector (Promega, USA), downstream of the luciferase gene, to generate the plasmids pGL3-WT-mTOR-3'UTR. pGL3-MUT-mTOR3'UTR was generated from pGL3-WT-mTOR-3'UTR by deleting the binding site for miR-99a "UACGGGU". For the luciferase reporter assay, the 786-0 and OS-RC-2 cell lines were co-transfected with luciferase reporter vectors and miR-99a mimics using Lipofectamine 2000. A 1-ng pRL-TK Renilla Luciferase construct was used for normalization. After 48 hours, luciferase activity was analyzed by the Dual-Luciferase Reporter Assay System according to the manufacturer's protocols (Promega, Madison, USA).

\section{Western blotting analysis}

Total protein was collected by Total Protein Extraction Kit (KeyGen, China); $30 \mu \mathrm{g}$ of protein per lane was separated by $12 \%$ SDS-polyacrylamide gel and transferred to PVDF membrane. The membrane was blocked in 5\% skim milk for 2 hours and then incubated with a specific antibody for 2 hours. The antibodies used in this study were: primary antibodies against Cyclin-D1, Cyclin-D2, Cyclin-E (Bioworld,Nanjing, China), mTOR, phosphomTOR, p70S6K, phospho-p70S6K, 4E-BP1 and phospho4E-BP1 (Cell Signaling Technology, USA). GAPDH and $\beta$-actin (Bioworld, Nanjing, China) on the same membrane was used as a loading control. The specific protein was detected by a BCA Protein Assay Kit (KeyGen, China). The band density of specific proteins was quantified after normalization with the density of GAPDH or $\beta$-actin.

\section{Statistical analysis}

Data are presented as the mean \pm standard deviation (SD) from at least three independent experiments. Student's $t$ test and one-way analysis of variance (ANOVA) were used to analyze significant differences using SPSS 17.0 (SPSS Inc., USA). All $P<0.05$ were marked with *, and $P<0.01$ with **. 


\section{Results}

miR-99a is downregulated and correlates with overall survival in renal cell carcinoma

To identify the expression of miR-99a in RCC, we firstly performed real-time $\mathrm{qRT}-\mathrm{PCR}$ using the renal cell line HK-2 and RCC cell lines 786-0 and OS-RC-2 and found that miR-99a expression in RCC cell lines (786-0 and OS-RC-2) was significantly lower than that in HK-2 (Figure 1A). Then we analysed miR-99a expression in clinical samples. Patient and tumor characteristics are showed in Table 1. Total RNA was extracted from 40 pairs of RCC and their adjacent non-tumor tissues and real-time qRT-PCR was performed. MiR-99a was considered to be significantly downregulated only if the calculated fold-change was less than 0.5 in the tumor tissue compared with the matched adjacent non-tumor tissue. As consistent with the results in cell lines, the expression of miR-99a was remarkably downregulated in RCC

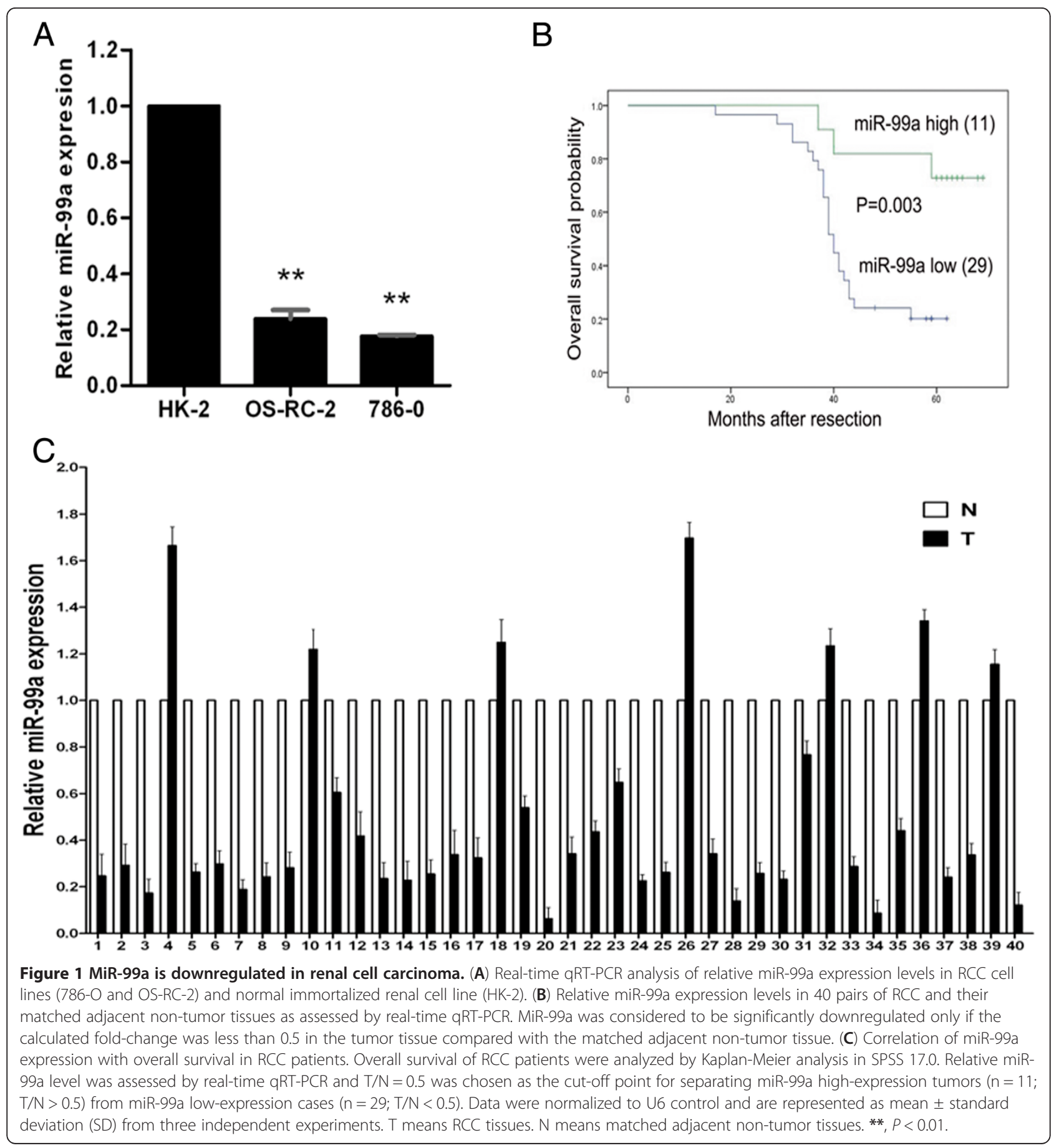


Table 1 Patients and tumor characteristics $(n=40$; 2005-2007)

\begin{tabular}{|c|c|c|c|c|c|}
\hline No. & Age & Sex & Pathologic Diagnosis & pT Stage & Grade \\
\hline 1 & 62 & female & clear cell & $T 1 b$ & 2 \\
\hline 2 & 61 & male & clear cell & T3b & 2 \\
\hline 3 & 64 & male & clear cell & T4 & 3 \\
\hline 4 & 69 & female & clear cell & T1a & 1 \\
\hline 5 & 73 & male & clear cell & $\mathrm{T} 2 \mathrm{~b}$ & 4 \\
\hline 6 & 62 & female & clear cell & Т3а & 3 \\
\hline 7 & 53 & male & clear cell & $\mathrm{T} 2 \mathrm{~b}$ & 2 \\
\hline 8 & 57 & male & clear cell & $\mathrm{T} 3 \mathrm{~b}$ & 3 \\
\hline 9 & 42 & male & clear cell & $\mathrm{T} 2 \mathrm{~b}$ & 3 \\
\hline 10 & 64 & male & clear cell & T2a & 2 \\
\hline 11 & 70 & female & clear cell & $\mathrm{T} 1 \mathrm{~b}$ & 2 \\
\hline 12 & 68 & female & clear cell & T3а & 3 \\
\hline 13 & 48 & male & clear cell & Т3а & 3 \\
\hline 14 & 78 & male & clear cell & $\mathrm{T} 1 \mathrm{~b}$ & 2 \\
\hline 15 & 65 & male & clear cell & Т3а & 3 \\
\hline 16 & 38 & female & clear cell & T3a & 2 \\
\hline 17 & 56 & male & clear cell & T3b & 3 \\
\hline 18 & 44 & female & clear cell & Т3а & 2 \\
\hline 19 & 63 & female & clear cell & T2a & 1 \\
\hline 20 & 69 & male & clear cell & T4 & 2 \\
\hline 21 & 55 & male & clear cell & T3b & 3 \\
\hline 22 & 60 & male & clear cell & Т3а & 2 \\
\hline 23 & 38 & female & clear cell & T1a & 1 \\
\hline 24 & 45 & male & clear cell & T3b & 2 \\
\hline 25 & 62 & female & clear cell & T3b & 2 \\
\hline 26 & 41 & male & clear cell & $\mathrm{T} 1 \mathrm{~b}$ & 3 \\
\hline 27 & 60 & female & clear cell & $\mathrm{T} 2 \mathrm{~b}$ & 3 \\
\hline 28 & 63 & male & clear cell & T3b & 2 \\
\hline 29 & 35 & male & clear cell & $\mathrm{T} 1 \mathrm{~b}$ & 4 \\
\hline 30 & 77 & male & clear cell & T3a & 3 \\
\hline 31 & 64 & male & clear cell & T2a & 3 \\
\hline 32 & 42 & female & clear cell & T1a & 1 \\
\hline 33 & 68 & female & clear cell & T3b & 3 \\
\hline 34 & 71 & male & clear cell & T4 & 4 \\
\hline 35 & 52 & male & clear cell & $\mathrm{T} 2 \mathrm{~b}$ & 2 \\
\hline 36 & 62 & female & clear cell & T3b & 2 \\
\hline 37 & 69 & male & clear cell & Т3а & 3 \\
\hline 38 & 73 & male & clear cell & T3b & 3 \\
\hline 39 & 50 & female & clear cell & T2a & 2 \\
\hline 40 & 38 & male & clear cell & T1a & 3 \\
\hline
\end{tabular}

tissues (29/40, 72.5\%), compared with matched adjacent non-tumor tissues (Figure 1B). Notably, dramatic downregulation of miR-99a was observed in 50\% (9/18) cases of low stage (pT1 + pT2) and 91\% (20/22) cases of high stage (pT3+pT4) RCC. These results indicate that miR-99a expression possibly correlates with pathologic stage of RCC. To investigate whether downregulation of miR-99a in RCC tissues correlated with overall survival of RCC patients, we performed statistical analysis with Kaplan-Meier method. As shown in Figure 1C, lower miR-99a expression level in RCC tissues dramatically correlated with decreased overall survival of RCC patients. These data suggest that miR-99a may be a predictor for prognosis of RCC patients.

\section{miR-99a suppresses tumorigenicity in vitro}

The reduced expression of miR-99a in RCC prompted us to identify whether miR-99a functions as a tumor suppressor. To investigate the function of miR-99a, we restored miR-99a in RCC cell lines. 786-0 and OS-RC-2 cells were transfected with miR-99a or $\mathrm{NC}$, and then functional assays were performed. CCK- 8 assay showed that mir-99a restoration was more potent than their NC transfectants in inhibiting the proliferation of RCC cells. (Figure 2A). As shown in Figure 2B, compared with NC transfectants, miR-99a-restored RCC cells displayed notably fewer and smaller colonies. Transwell migration and invasion assays showed that the migration (Figure $2 \mathrm{C}$ ) and invasion (Figure 2D) of miR-99a-restored RCC cells were reduced compared with their NC transfectants, respectively. These observations suggest that miR-99a restoration suppresses the tumorigenicity of RCC cells in vitro.

\section{miR-99a induces G1-phase cell cycle arrest}

To investigate the role of miR-99a in cell cycle progression, we restored miR-99a in RCC cells. 786-O and OSRC-2 cells were transfected with miR-99a or NC. Cell cycle assay showed that mir-99a-restored RCC cells had a significant increase in G1-phase population as compared with NC transfectants (Figure 3A, B). Additionally, we also examined the effect of miR-99a on apoptosis and found that miR-99a restoration could hardly influence apoptosis in RCC cell lines (data not shown). These findings indicate that miR-99a induces G1-phase cell cycle arrest in RCC cell lines.

\section{miR-99a suppresses tumor growth in vivo}

Because the in vitro data demonstrated that miR-99a harbored antitumorigenic properties in RCC, we conducted a proof-of-principle experiment, in which a 786-0 xenograft model was used to confirm the effect of miR-99a on tumorigenicity in vivo. As shown in Figure 4A, twenty-five days following 786-0 cells subcutaneous inoculation, the mean tumor volume of the mice in the control and treated groups was 98 and $100 \mathrm{~mm}^{3}$, respectively. Then, miR-99a or NC mimics was repeatedly administered by intratumoral injections every 3 days for 4 weeks. At the end of 


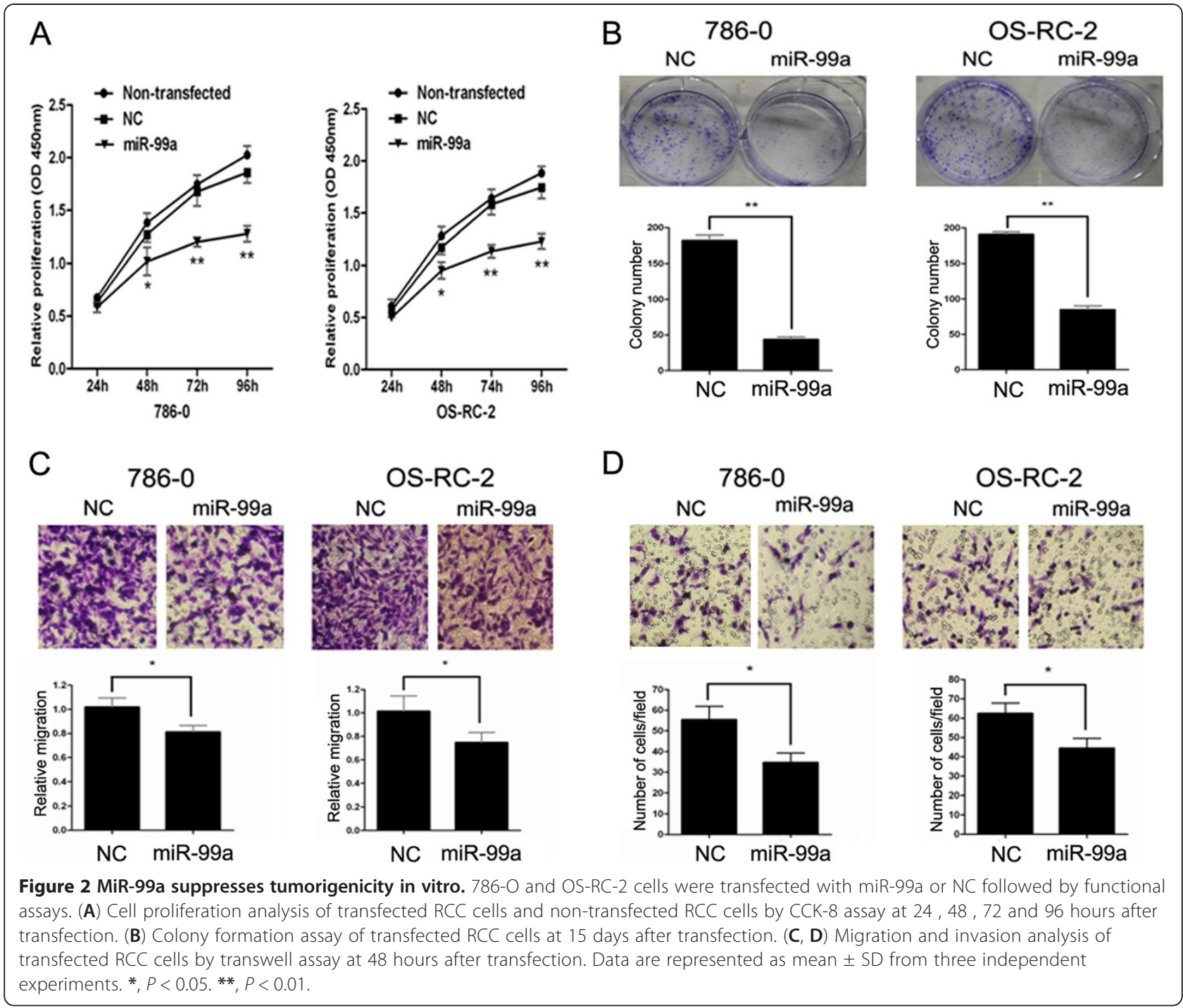

the experiment, intratumoral delivery of synthetic miR99a induced a specific inhibitory response and robustly interfered with tumor growth compared with control mice. In addition, We detected the expression of mTOR in tumor xenografts after miR-99a injection by Western blot, we found that intratumoral delivery of synthetic miR-99a makedly suppressed mTOR expression compared with control mice (Figure 4B). These results suggest that restoration of miR-99a suppresses tumor growth in vivo and could serve as a therapeutic tool in RCC therapy.

\section{mTOR is a target of miR-99a}

To explore the mechanisms by which miR-99a regulates the tumorigenicity of RCC, we performed a bioinformatic search (Targetscan, Pictar and MICROCOSM) for putative targets of miR-99a and found 3'UTR of mTOR containing the highly conserved putative miR-99a binding sites (Figure 5A). As mentioned above, miR-99a was remarkably downregulated in RCC cell lines (Figure 1A). Western blotting analysis found a clear upregulation of mTOR protein in RCC cell lines compared with HK-2 (Figure 5B). So, there was an inverse correlation between miR-99a levels and mTOR protein. To show that miR-99a participated in the regulation of mTOR expression, we restored miR-99a in RCC cells. 786-0 and OS-RC-2 cells were transfected with miR-99a or NC. The enforced expression of miR-99a in RCC cell lines led to a decrease in mTOR protein and also led to a decrease in phosphomTOR (p-mTOR) protein, compared with NC transfectants (Figure 5C). To ascertain the direct miR-99a-mTOR interaction, we created pGL3-WT-mTOR-3'UTR and pGL3-MUT- mTOR-3'UTR plasmids. Dual-luciferase reporter assay revealed that restoration of miR-99a led to a marked decrease in luciferase activity of pGL3-WT-mTOR$3^{\prime}$ UTR plasmid in 786-0 and OS-RC-2 cells but did not change luciferase activity of pGL3-MUT- mTOR-3'UTR 


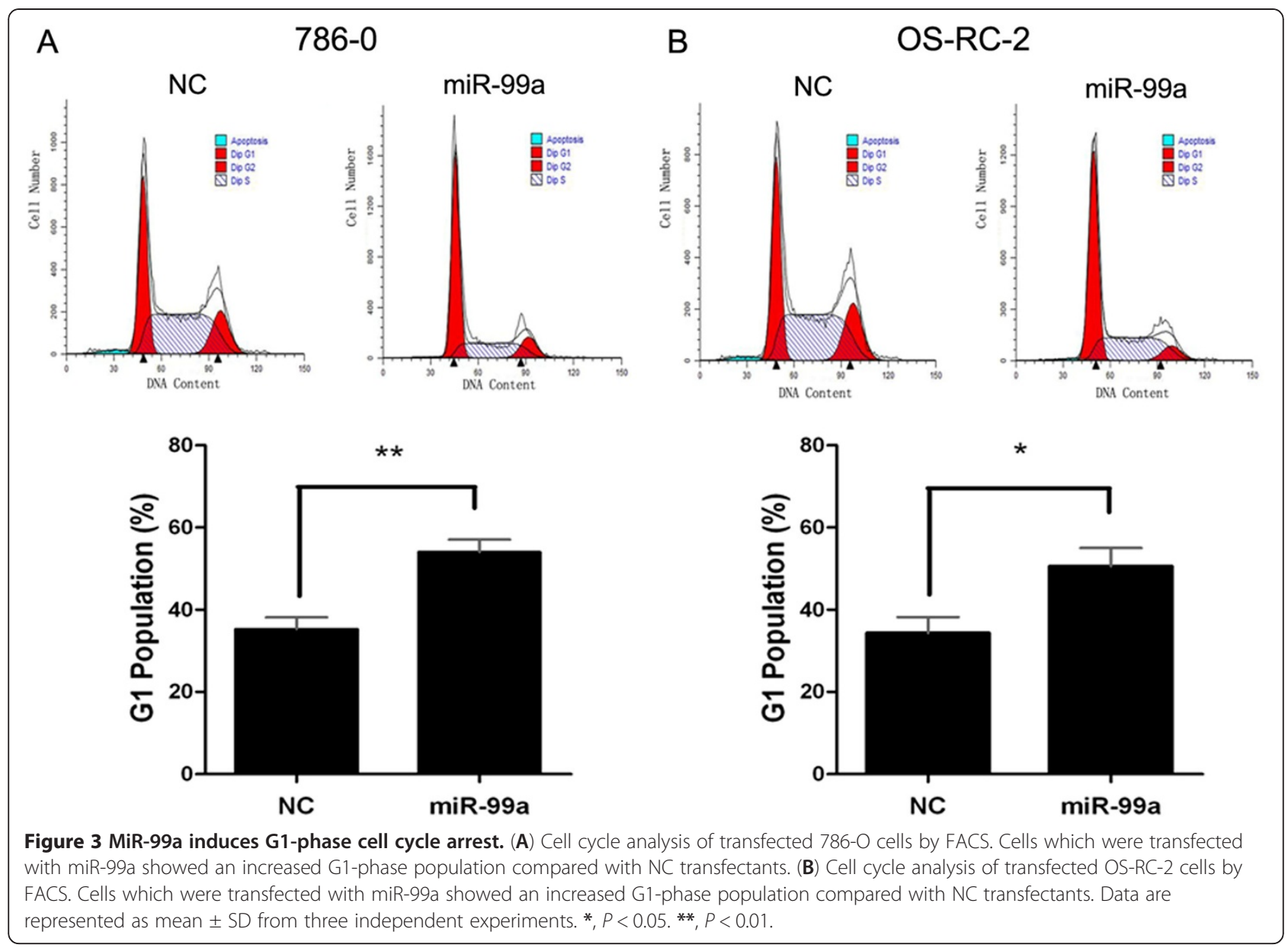

(Figure 5D). Taken together, these findings showed a direct interaction between miR-99a and mTOR mRNA in RCC cell lines.

\section{mTOR pathway is involved in miR-99a mediated G1/S} transition

To evaluate whether mTOR pathway is implicated in miR-99a induced G1-phase arrest, downstream substrates of mTOR pathway were investigated after restoration of miR-99a in 786-0 cells. We detected ribosomal protein S6 kinase, 70 kDa (P70S6K), phospho-p70S6K (p-p70S6K), Eukaryotic translation initiation factor 4E-binding protein 1 (4E-BP1) and phospho-4E-BP1 (p-4E-BP1) expression by western blotting analysis. As shown in Figure 6A, compared with NC transfectants, the expression of p-p70S6K and p-4E-BP1 were downregulated in

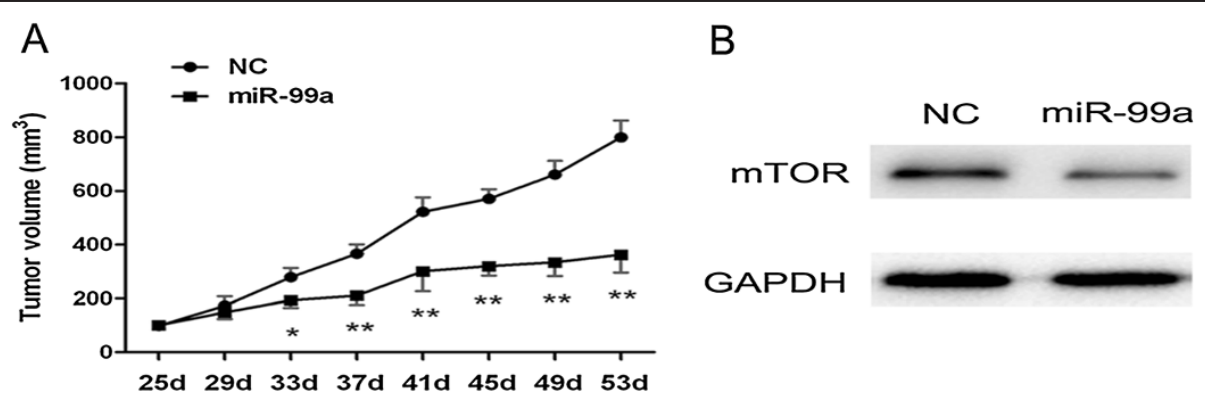

Figure 4 MiR-99a suppresses tumor growth in vivo. (A) 786-O cells were subcutaneously injected into nude mice to form solid, palapable tumors (day 25), following which synthetic miR-99a or NC mimics were intratumorally delivered for 4 weeks. Tumor volumes following miR-99a administration were significantly reduced compared with the control mice. (B) After tumor xenografts were intratumorally delivered synthetic miR-99a or NC mimics for 4 weeks, we extraced the protein and performed Western blot. We found that intratumoral delivery of synthetic miR99a induced a makedly inhibition of mTOR expression compared with control mice. Data are represented as mean \pm SD. ${ }^{*}, P<0.05 .{ }^{*}, P<0.01$. 


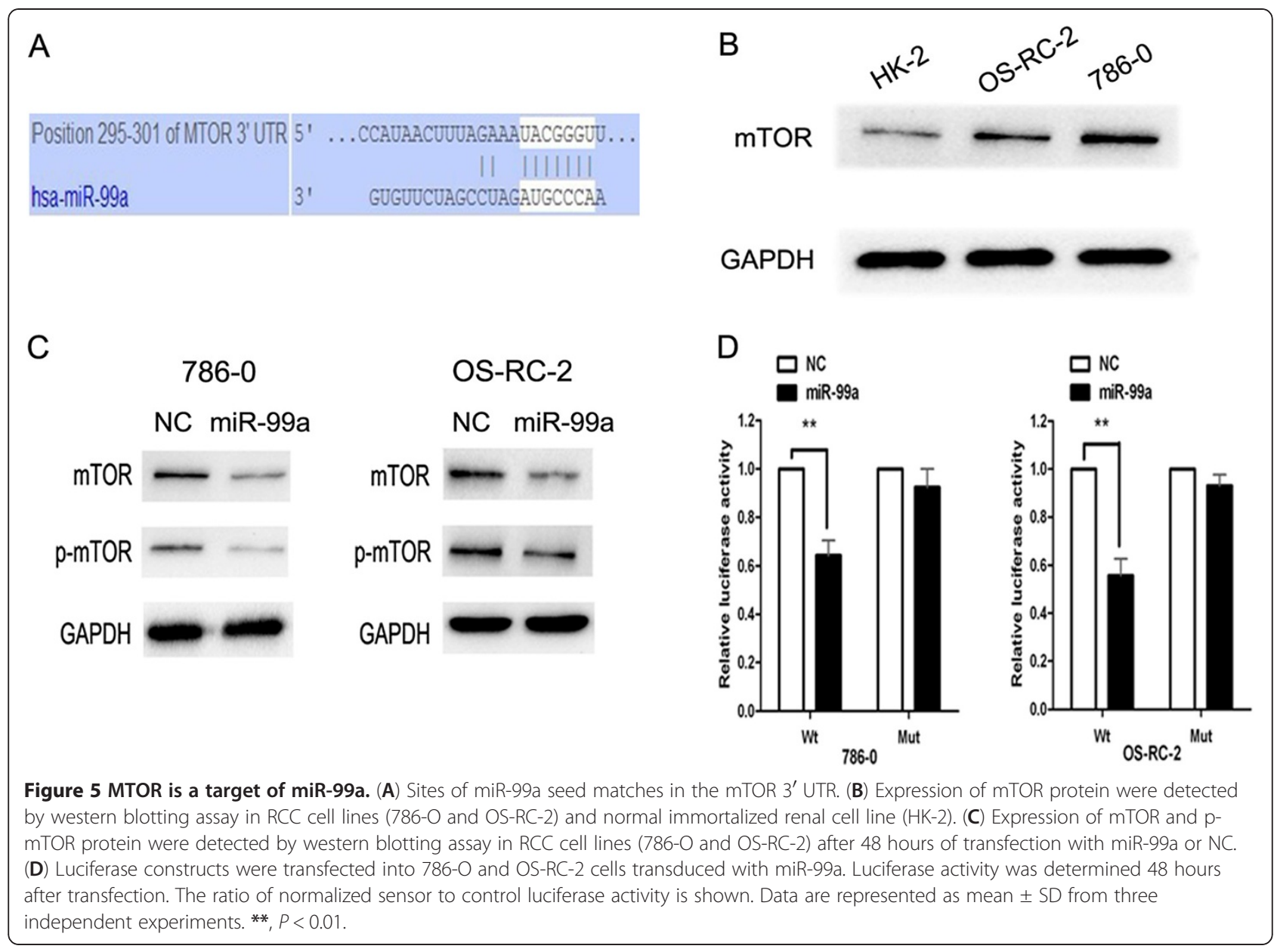

miR-99a-restored 786-0 cells, which suppressed the activation of sequential signaling cascades involved in synthesis of several G1/S transition-related molecules [21,22]. Then we detected the expression of cyclin D1, cyclin D3 and cyclin E in miR-99a-restored 786-0 cells. Western blotting analysis showed that cyclin D1, cyclin D3 and cyclin E expression were also downregulated (Figure 6B), which may be attributed to attenuated p-P70S6K and $\mathrm{p}-4 \mathrm{E}-\mathrm{BP} 1$. These results demonstrate that mTOR pathway is involved in miR-99a mediated G1/S Transition.

\section{mTOR knockdown partially phenocopies miR-99a} restoration in renal cell carcinoma cells

To further reveal mechanisms underlying this tumor suppressive effect of miR-99a, we knockdowned mTOR in RCC cells. 786-0 cells were transfected with mTORsiRNA or $\mathrm{NC}$, and then functional assays were performed. As expected, compared with NC transfectants, mTOR-knockdowned 786-0 cells showed a decrease in the proliferation and colony formation and an increase in the G1-phase population (Figure 7A-C), similar to the phenotype observed upon miR-99a restoration in 786-0 cells. However, the migration and invasion of
mTOR- knockdowned 786-0 cells were not decreased compared with NC transfectants (Figure 7D, E), which suggests that the regulation of miR-99a on migration and invasion in RCC cells is not likely related to mTOR inhibition. Taken together, we conclude that the tumor

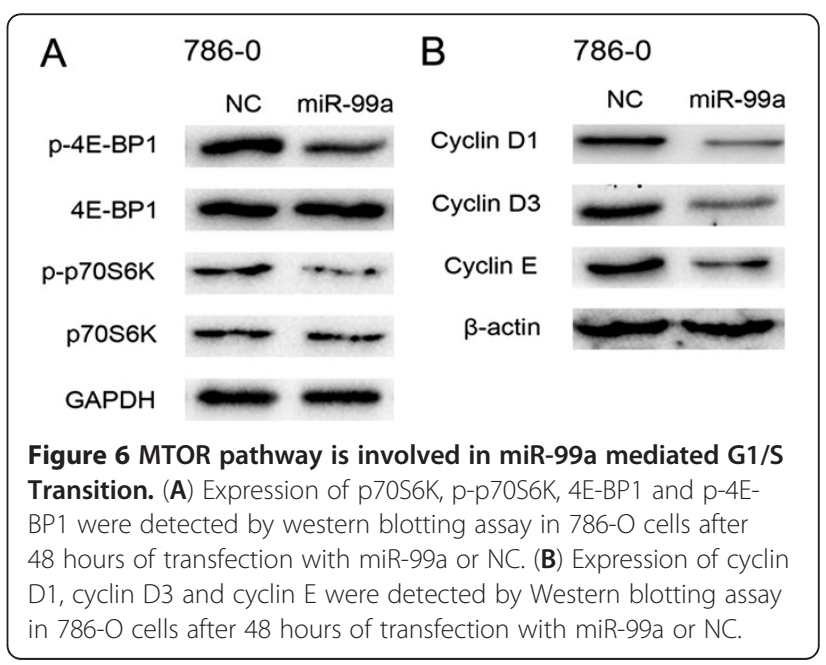




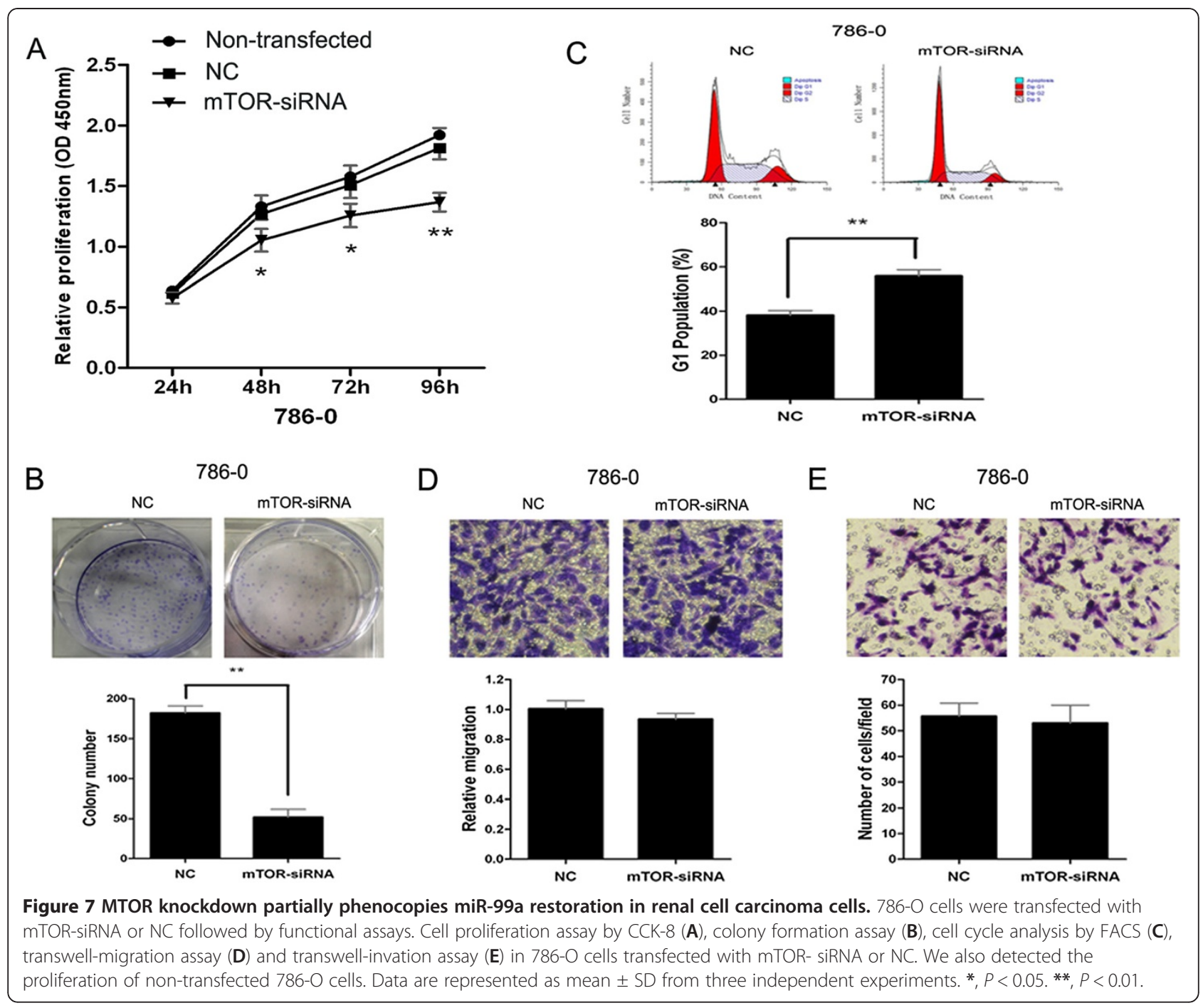

suppressive role of miR-99a may be mediated partially through mTOR pathway regulation.

\section{Discussion}

Previous studies have reported that miR-99a participated in tumorigenesis of several tumor type,including hepatocellular carcinoma [17], prostate cancer [19], childhood adrenocortical tumors [20] and lung cancer [23]. However, in this study, we demonstrate for the first time that miR-99a is implicated in the carcinogenesis of RCC. Compared with nonmalignant immortalized renal cell line HK-2, the expression of miR-99a was significantly downregulated in RCC cell lines 786-0 and OS-RC-2. As consistent with the results in cell lines, detection of miR-99a in RCC tissues also pointed to a dramatic attenuation of miR-99a expression in $72.5 \%$ (29/40) of RCC tissues. Notably, dramatic downregulation of miR-99a was observed in 50\% (9/18) cases of low stage (pT1 + pT2) and 91\% (20/22) cases of high stage
(pT3 + pT4) RCC. In addition, lower miR-99a expression level in RCC tissues significantly correlated with reduced overall survival in RCC patients. These results indicate that miR-99a may serve as a potential predictor for prognosis of RCC patients. A limitation to our study was the relatively small number of clinical samples at our disposal. Further studies with more clinical samples are warranted.

The reduced expression of miR-99a in RCC prompted us to identify whether miR-99a functions as a tumor suppressor. We found that restoration of miR-99a suppressed cell growth, clonability, migration and invasion and induced G1-phase cell cycle arrest in vitro. Moreover, intratumoral delivery of miR-99a was sufficient to trigger in vivo regression of tumor growth in RCC xenograft model. These findings suggest that miR-99a plays a tumor suppressive role and may be a therapeutic intervention in RCC. It has been reported that overexpression of miR-99a inhibits the growth of prostate cancer 
cells and decreases the expression of prostate-specific antigen (PSA) [19]. In addition, restoration of miR-99a dramatically suppresses tumor cell growth in lung cancer [23]. Recently, Li et al. reported that restoration of miR-99a significantly inhibits hepatocellular carcinoma cell growth in vitro by inducing the G1 phase cell cycle arrest [17]. All these reports support our findings in RCC. However, Li et al. also reported that restoration of miR-99a could hardly influence the metastasis of hepatocellular carcinoma cell lines [17], inconsistent with our findings in RCC. Although the actual reasons are currently unclear, this inconsistency might be due to the different tumor type and cellular context.

With the help of bioinformatics prediction and sequential experimental demonstration, mTOR was identified as a direct target of miR-99a in RCC. MTOR signaling pathway is a key signal-transduction system that links multiple receptors and oncogenic molecules to diverse cellular functions and is inappropriately activated in many human cancers $[24,25]$. MTOR signaling pathway plays a crucial role in the regulation of cell growth, protein translation, metabolism, cell invasion, and cell cycle [26]. Major downstream targets of mTOR are p70S6K and 4E-BP1, which is activated by mTOR and then dissociates from the eukaryotic translation factor (eIF-4E) and activates protein synthesis [27]. Overexpression or overactivation of mTOR may strengthen the signals passed down by mTOR signaling pathway, which will cause over-phosphorylation of the downstream molecules p70S6K and 4E-BP1. Once phosphorylated, p70S6K and 4E-BP1 can promote protein synthesis [17]. Thus, several cell-cycle related proteins including cyclin D1, cyclin D3 and cyclin E [21,22], will be excessively upregulated which resulted in the progression of cell cycle. We restored miR-99a in 786-0 cells and found that the expression of p-p70S6K, p-4E-BP1, cyclin D1, cyclin D3 and cyclin $\mathrm{E}$ are really downregulated, consistent with the previous reports in hepatocellular carcinoma [17]. Therefore, activation of the mTOR pathway provides tumor cells with a growth advantage by promoting protein synthesis [28].

To further elucidate mechanisms underlying the tumor suppressive effect of miR-99a, we knockdowned mTOR in 786-0 cells and found that the proliferation and colony formation were decreased and the G1-phase population was increased, similar to the phenotype observed upon miR-99a restoration in 786-0 cells. However, the migration and invasion of mTOR-knockdowned 786-0 cells were not decreased, which suggests that the regulation of miR-99a on migration and invasion in RCC cells is not likely related to mTOR inhibition. There results suggest that the tumor suppressive role of miR-99a may be mediated partially through mTOR pathway regulation.

On the basis of these findings, we propose a hypothetical model for the function of the miR-99a-mTOR axis in RCC. Downregulation of miR-99a leading to increase of $\mathrm{mTOR}$ and $\mathrm{p}-\mathrm{mTOR}$ results in the phosphorylation of $4 \mathrm{E}-\mathrm{BP} 1$ and $\mathrm{p} 70 \mathrm{~S} 6 \mathrm{~K}$, which in turn activates protein synthesis,promotes cell proliferation and cell clonability and allows progression from the G1 to the S phase of the cell cycle. It has been reported that miR-100 is downregulated and targets mTOR in clear cell ovarian cancer [29] and childhood adrenocortical tumors [20]. More recently, miR-199a-3p was also shown to be downregulated and target $\mathrm{mTOR}$ in hepatocarcinoma cells [30]. These characteristics of miR-100 and miR199a-3p are quite similar to those of miR-99a, indicating that mTOR expression might be regulated redundantly by various closely related miRNAs. It is postulated that each miRNA regulates up to 100 different mRNAs and that more than 10,000 mRNAs appear to be directly regulated by miRNAs [31]. In our study, we found that the regulation of miR-99a on migration and invasion in RCC cells is not likely related to mTOR inhibition. Thus, it remains possible other targets might be at least partially involved. The mechanisms underlying miR-99a implicated in the carcinogenesis of RCC is very complicated, and further extensive analysis will be necessary to elucidate the precise mechanisms of miR-99a implicated in the carcinogenesis of RCC.

Expression of miR-99a has been proved frequently downregulated in various tumors [14-20], but the mechanisms underlying the downregulation of miR-99a in cancers remain to be unknown. It has been reported that downregulation of miR-99a is caused by the activation of Src/Rasrelated pathways in human tumors [23]. The gene encoding miR-99a was found residing within an intron of C21or $\mathrm{f} 34, \mathrm{C} 21$ or $\mathrm{f} 34$ located in chromosome 21q21, the region was commonly deleted in lung cancer [13,32]. Recently, miR-99a was also shown to be co-transcripted with C21 or f34 in hepatocellular carcinoma [17]. Up to date, there are no studies on the mechanisms of miR-99a downregulation in RCC, so illuminating the mechanisms responsible for downregulation of miR-99a in RCC would be our next study in the future.

\section{Conclusions}

In conclusion, our study demonstrates for the first time that deregulation of miR-99a is involved in the etiology of RCC partially via direct targeting mTOR pathway. In view of our present results showing decreased miR-99a expression in RCC clinical samples correlating with overall survival of RCC patients and the suppression of tumorigenicity upon upregulation of miR-99a in vitro and in vivo, we propose a hypothesis that miR-99a may be an attractive target for prognostic and therapeutic interventions in RCC.

Competing interests

We declare that we have no conflict of interest. 


\section{Authors' contributions}

$\mathrm{LC}, \mathrm{HZ}$ and $\mathrm{HZ}$ carried out the experimental studies and performed the statistical analysis. LC drafted and completed the manuscript. YZ was in charge of the clinical samples selection and performed the proofreading. RX, $X X, L Z, Z X, W X$ and BZ disposed the tissue samples. TD and YC completed sample conservation. ZT and QS refined the manuscript. XH conceived and designed of the study. All authors read and approved the final manuscript.

\section{Acknowledgments}

This work was supported by the National Natural Science Foundation (No.81273267) and the Natural Science Foundation of Jiangsu province (BK2011248)

\section{Author details}

'Department of Urology, The Third Affiliated Hospital of Soochow University, 185 Juqian Street, Changzhou 213003, China. ${ }^{2}$ Department of Nephrology, The Third Affiliated Hospital of Soochow University, Changzhou, China. ${ }^{3}$ Department of Urology, The Affiliated Jiangyin Hospital of Southeast University Medical College, Wuxi, China. ${ }^{4}$ Comprehensive Laboratory, The Third Affiliated Hospital of Soochow University, Changzhou, China.

Received: 31 August 2012 Accepted: 19 November 2012

Published: 23 November 2012

\section{References}

1. White NM, Yousef GM: MicroRNAs: exploring a new dimension in the pathogenesis of kidney cancer. BMC Med 2010, 8:65.

2. van Spronsen DJ, de Weijer KJ, Mulders PF, De Mulder PH: Novel treatment strategies in clear-cell metastatic renal cell carcinoma. Anticancer Drugs 2005, 16(7):709-717.

3. Pascual D, Borque A: Epidemiology of kidney cancer. Adv Urol 2008, 2008:782381.

4. Reeves DJ, Liu CY: Treatment of metastatic renal cell carcinoma. Cancer Chemother Pharmacol 2009, 64(1):11-25.

5. Janzen NK, Kim HL, Figlin RA, Belldegrun AS: Surveillance after radical or partial nephrectomy for localized renal cell carcinoma and management of recurrent disease. Urol Clin North Am 2003, 30(4):843-852.

6. Chow TF, Youssef YM, Lianidou E, Romaschin AD, Honey RJ, Stewart R, Pace $K T$, Yousef GM: Differential expression profiling of microRNAs and their potential involvement in renal cell carcinoma pathogenesis. Clin Biochem 2010, 43(1-2):150-158.

7. Garzon R, Pichiorri F, Palumbo T, Visentini M, Aqeilan R, Cimmino A, Wang $H$, Sun $H$, Volinia $S$, Alder $H$, et al: MicroRNA gene expression during retinoic acid-induced differentiation of human acute promyelocytic leukemia. Oncogene 2007, 26(28):4148-4157.

8. Schickel R, Boyerinas B, Park SM, Peter ME: MicroRNAs: key players in the immune system, differentiation, tumorigenesis and cell death. Oncogene 2008, 27(45):5959-5974.

9. Calin GA, Croce CM: MicroRNA signatures in human cancers. Nat Rev Cancer 2006, 6(11):857-866.

10. Huang Y, Dai Y, Yang J, Chen T, Yin Y, Tang M, Hu C, Zhang L: Microarray analysis of microRNA expression in renal clear cell carcinoma. Eur I Surg Oncol 2009, 35(10):1119-1123.

11. Shenouda SK, Alahari SK: MicroRNA function in cancer: oncogene or a tumor suppressor? Cancer Metastasis Rev 2009, 28(3-4):369-378.

12. Iorio MV, Croce CM: MicroRNAs in cancer: small molecules with a huge impact. J Clin Oncol 2009, 27(34):5848-5856.

13. Nagayama K, Kohno T, Sato M, Arai Y, Minna JD, Yokota J: Homozygous deletion scanning of the lung cancer genome at a $100-\mathrm{kb}$ resolution. Genes Chromosomes Cancer 2007, 46(11):1000-1010.

14. Nam EJ, Yoon H, Kim SW, Kim H, Kim YT, Kim JH, Kim JW, Kim S: MicroRNA expression profiles in serous ovarian carcinoma. Clin Cancer Res 2008, 14(9):2690-2695

15. Wong TS, Liu XB, Wong BY, Ng RW, Yuen AP, Wei WI: Mature miR-184 as potential oncogenic microRNA of squamous cell carcinoma of tongue. Clin Cancer Res 2008, 14(9):2588-2592.

16. Gao W, Shen H, Liu L, Xu J, Xu J, Shu Y: MiR-21 overexpression in human primary squamous cell lung carcinoma is associated with poor patient prognosis. J Cancer Res Clin Oncol 2011, 137(4):557-566.

17. Li D, Liu X, Lin L, Hou J, Li N, Wang C, Wang P, Zhang Q, Zhang P, Zhou W, et al: MicroRNA-99a inhibits hepatocellular carcinoma growth and correlates with prognosis of patients with hepatocellular carcinoma. $J$ Biol Chem 2011, 286(42):36677-36685.

18. Catto JW, Miah S, Owen HC, Bryant H, Myers K, Dudziec E, Larre S, Milo M, Rehman I, Rosario DJ, et al: Distinct microRNA alterations characterize high- and low-grade bladder cancer. Cancer Res 2009, 69(21):8472-8481.

19. Sun D, Lee YS, Malhotra A, Kim HK, Matecic M, Evans C, Jensen RV, Moskaluk CA, Dutta A: miR-99 family of microRNAs suppresses the expression of prostate-specific antigen and prostate cancer cell proliferation. Cancer Res 2011, 71(4):1313-1324.

20. Doghman M, El WA, Cardinaud B, Thomas E, Wang J, Zhao W, Peralta-Del VM, Figueiredo BC, Zambetti GP, Lalli E: Regulation of insulin-like growth factor-mammalian target of rapamycin signaling by microRNA in childhood adrenocortical tumors. Cancer Res 2010, 70(11):4666-4675.

21. Fingar DC, Richardson CJ, Tee AR, Cheatham L, Tsou C, Blenis J: mTOR controls cell cycle progression through its cell growth effectors S6K1 and 4E-BP1/eukaryotic translation initiation factor 4E. Mol Cell Biol 2004, 24(1):200-216

22. Grewe M, Gansauge F, Schmid RM, Adler G, Seufferlein T: Regulation of cell growth and cyclin D1 expression by the constitutively active FRAPp70s6K pathway in human pancreatic cancer cells. Cancer Res 1999, 59 (15):3581-3587.

23. Oneyama C, Ikeda J, Okuzaki D, Suzuki K, Kanou T, Shintani Y, Morii E, Okumura M, Aozasa K, Okada M: MicroRNA-mediated downregulation of mTOR/FGFR3 controls tumor growth induced by Src-related oncogenic pathways. Oncogene 2011, 30(32):3489-3501.

24. Petroulakis E, Mamane Y, Le Bacquer O, Shahbazian D, Sonenberg N: mTOR signaling: implications for cancer and anticancer therapy. $\mathrm{Br} J$ Cancer 2007, 96(Suppl):R11-R15.

25. Guertin DA, Sabatini DM: Defining the role of mTOR in cancer. Cancer Cell 2007, 12(1):9-22.

26. Vignot S, Faivre S, Aguirre D, Raymond E: mTOR-targeted therapy of cancer with rapamycin derivatives. Ann Oncol 2005, 16(4):525-537.

27. Kapoor A: Inhibition of mTOR in kidney cancer. Curr Oncol 2009, 16(Suppl 1):S33-S39.

28. Menon S, Manning BD: Common corruption of the mTOR signaling network in human tumors. Oncogene 2008, 27(Suppl 2):S43-S51.

29. Nagaraja AK, Creighton CJ, Yu Z, Zhu H, Gunaratne PH, Reid JG, Olokpa E, Itamochi H, Ueno NT, Hawkins SM, et al: A link between mir-100 and FRAP1/mTOR in clear cell ovarian cancer. Mol Endocrinol 2010, 24(2):447-463.

30. Fornari F, Milazzo M, Chieco P, Negrini M, Calin GA, Grazi GL, Pollutri D, Croce CM, Bolondi L, Gramantieri L: MiR-199a-3p regulates mTOR and cMet to influence the doxorubicin sensitivity of human hepatocarcinoma cells. Cancer Res 2010, 70(12):5184-5193.

31. Hummel R, Hussey DJ, Haier J: MicroRNAs: predictors and modifiers of chemo- and radiotherapy in different tumour types. Eur J Cancer 2010 46(2):298-311

32. Yamada H, Yanagisawa K, Tokumaru S, Taguchi A, Nimura Y, Osada H, Nagino M, Takahashi T: Detailed characterization of a homozygously deleted region corresponding to a candidate tumor suppressor locus at 21q11-21 in human lung cancer. Genes Chromosomes Cancer 2008, 47(9):810-818

doi:10.1186/1471-2407-12-546

Cite this article as: Cui et al:: MicroRNA-99a induces G1-phase cell cycle arrest and suppresses tumorigenicity in renal cell carcinoma. BMC Cancer 2012 12:546. 